\title{
Chronic medication does not affect hyperactive error responses in obsessive-compulsive disorder
}

\author{
EMILY R. STERN, ${ }^{\mathrm{a}}$ YANNI LIU, ${ }^{\mathrm{b}}$ WILLIAM J. GEHRING,${ }^{\mathrm{b}}$ JAMES J. LISTER, ${ }^{\mathrm{a}}$ GANG YIN,,${ }^{\mathrm{b}, \mathrm{c}}$ \\ JUN ZHANG, ${ }^{\text {b }}$ KATE D. FITZGERALD, ${ }^{\mathrm{a}}$ JOSEPH A. HIMLE, ${ }^{\mathrm{a}}{ }^{\mathrm{J}} \mathrm{AMES}$ L. ABELSON, ${ }^{\mathrm{a}}$ AND \\ STEPHAN F. TAYLOR ${ }^{\mathrm{a}}$ \\ ${ }^{a}$ Department of Psychiatry, University of Michigan, Ann Arbor, Michigan, USA \\ ${ }^{\mathrm{b}}$ Department of Psychology, University of Michigan, Ann Arbor, Michigan, USA \\ ${ }^{\mathrm{c}}$ Center for Neuroinformatics, School of Life Science and Technology, University of Electronic Science and Technology of China, Chengdu, China
}

\begin{abstract}
Patients with obsessive-compulsive disorder (OCD) show an increased error-related negativity (ERN), yet previous studies have not controlled for medication use, which may be important given evidence linking performance monitoring to neurotransmitter systems targeted by treatment, such as serotonin. In an examination of 19 unmedicated OCD patients, 19 medicated OCD patients, 19 medicated patient controls without OCD, and 21 unmedicated healthy controls, we found greater ERNs in OCD patients than in controls, irrespective of medication use. Severity of generalized anxiety and depression was associated with ERN amplitude in controls but not patients. These data confirm previous findings of an exaggerated error response in OCD, further showing that it cannot be attributed to medication. The absence in patients of a relationship between ERN amplitude and anxiety/depression, as was found in controls, suggests that elevated error signals in OCD may be disorder-specific.
\end{abstract}

Descriptors: Error-related negativity (ERN), Event-related potentials (ERPs), Anxiety, Anterior cingulate cortex (ACC)

Obsessive-compulsive disorder (OCD) is characterized by intrusive thoughts (obsessions) and/or repetitive behaviors (compulsions) that are often associated with intense doubt regarding the correctness of an act or excessive fear about the likelihood of a bad outcome. Proposals that symptoms are related to an overactive error detection mechanism that continually signals that "something is wrong" (Pitman, 1987; Schwartz, 1997) have been supported by research identifying alterations in the neural substrate for error processing in OCD. Errors elicit robust activation in a network of brain regions including medial frontal cortex (MFC) (Taylor, Stern, \& Gehring, 2007). In particular, the errorrelated negativity (ERN, or Ne) component of the event-related brain potential (ERP), which onsets at the time of error commission (Falkenstein, Hohnsbein, Hoormann, \& Blanke, 1990; Gehring, Goss, Coles, Meyer, \& Donchin, 1993) and localizes to MFC (Dehaene, Posner, \& Tucker, 1994; van Veen \& Carter, 2002), has emerged as an index of dysfunctional error processing in OCD. Several groups have found an increased ERN in patients with clinical OCD (Endrass, Klawohn, Schuster, \& Kathmann, 2008; Gehring, Himle, \& Nisenson, 2000; Johannes

Support for this research has been provided by the National Institute of Mental Health (NIMH) R01 MH071821 awarded to SFT and F32 MH082573 awarded to ERS.

Address reprint requests to: Emily R. Stern, Ph.D., University of Michigan, 4250 Plymouth Road, 2506 Rachel Upjohn Building, Ann Arbor, MI 48106. E-mail: emistern@med.umich.edu et al., 2001; but see Nieuwenhuis, Nielen, Mol, Hajcak, \& Veltman, 2005 for alternate finding) and undiagnosed subjects with high ratings of OC symptoms (Hajcak \& Simons, 2002).

While the error signal appears to be increased in OCD, previous studies have not always controlled for other factors that may influence the ERN. In particular, medications such as serotonin reuptake inhibitors (SRIs) are commonly used to treat patients with OCD, and many studies of error processing in OCD have tested groups where some or all patients were taking medication (Endrass et al., 2008; Gehring et al., 2000; Nieuwenhuis et al., 2005; Ursu, Stenger, Shear, Jones, \& Carter, 2003). While the influence of dopamine in generating and/or modulating the ERN has perhaps received the most attention (Holroyd \& Coles, 2002), evidence now also links serotonergic (Fallgatter et al., 2004) and noradrenergic systems to performance monitoring (for a review, see Jocham \& Ullsperger, 2009), pointing to the need to consider medication status when investigating the ERN in psychiatric populations. A very few studies have examined unmedicated patients, but in small samples. Johannes and colleagues (2001) found increased ERNs in 10 unmedicated OCD patients compared to healthy controls, suggesting that a hyperactive ERN is not related to medication. In a direct comparison of medicated and unmedicated OCD subjects (Endrass et al., 2008), the ERN was significantly lower in patients taking medication, but it is possible that this effect was due to differences in illness severity between the groups. Given that severely ill patients are more likely to be on medication, disentangling the potential 
confound between medication use and illness severity remains a methodological challenge.

In order to examine the effects of chronic medication on error responses in OCD, we examined ERNs in OCD patients free of psychotropic medication, OCD patients on SRIs, patient controls on SRIs but without OCD, and healthy control subjects free of psychotropic medication and psychiatric illness. Any effect of medication on the ERN that is unrelated to OCD would be expected to be found in both medicated OCD patients and patient controls, but absent in unmedicated OCD patients and healthy controls.

\section{Methods}

\section{Subjects}

Eighty subjects performed the task while electroencephalogram (EEG) data were acquired. However, two OCD patients were excluded due to performance on the task; the amount of errors exhibited by one patient was over 3 standard deviations from the entire group mean ( $>180$ errors) and another made fewer than 10 errors. Due to a recent study reporting high internal reliability of the ERN with 10 errors and moderate reliability with 6 errors (Olvet \& Hajcak, 2009), we also performed all analyses reported below with the inclusion of the subject who made under 10 errors, and found results to be unchanged. The final group of 78 subjects were distributed as follows: 1) 19 OCD patients who were unmedicated (uOCD), 2) 19 OCD patients taking medication (mOCD), all of whom were on at least one serotonin reuptake inhibitor (with the exception of one patient who was taking buproprion), 3) 19 medicated patient controls (mPC), all of whom were taking at least one serotonin reuptake inhibitor due to prior history of major depression (in full or partial remission, based on DSM-IV criteria), and 4) 21 healthy controls (uHC) free of psy- chotropic medication and without current or past psychiatric diagnoses. Demographic and clinical variables of interest were examined for each group, as shown in Table 1. Unmedicated OCD and uHC groups were age- and education-matched, as were $\mathrm{mOCD}$ and $\mathrm{mPC}$ groups. Group differences in age, years of education, and scores from the Hamilton Anxiety Rating Scale (HARS) and Hamilton Depression Rating Scale (HDRS) were evaluated with separate $2 \times 2$ ANOVAs using diagnosis (OCD vs. control) and medication (unmedicated vs. medicated) as between-subjects factors. Chi-square tests compared gender (all groups), the proportion of subjects with a history of depression (uOCD, mOCD, and mPC groups), the proportion in current treatment for OCD (uOCD and mOCD groups), and the proportion with a history of hospitalizations for OCD (uOCD and mOCD groups). Independent samples $t$-tests were used to compare $\mathrm{uOCD}$ and $\mathrm{mOCD}$ groups on Yale-Brown Obsessive-Compulsive Scale (YBOCS) scores (Goodman et al., 1989), age of onset of OCD, and illness duration.

Subjects were recruited through paper advertisements posted around the local community, online advertisements through the University of Michigan Health System, and, for OCD patients, from referrals from the Anxiety Disorders Unit of the University of Michigan Depression Center/Ambulatory Psychiatry clinics. Patients in both OCD groups met DSM-IV criteria for primary diagnoses of OCD and were free of comorbid psychiatric disorders with the exception of tic disorder and/or specific phobia. Patients with hoarding as a primary symptom were excluded. OCD patients were accepted if they had current depressive disorder not otherwise specified (NOS) or had histories of major depressive disorder or dysthymia but were in partial or full remission from their depression $(79 \%$ of mOCD patients and $53 \%$ of uOCD patients). While all 19 medicated OCD patients were in treatment for OCD, with 15 out of the 19 being seen in our Anxiety clinic, many unmedicated OCD patients (13/19) were recruited through advertisements and were not in any treatment

Table 1. Demographic Information

\begin{tabular}{|c|c|c|c|c|c|c|c|}
\hline & $\mathrm{uOCD}(n=19)$ & $\operatorname{mOCD}(n=19)$ & $\operatorname{mPC}(n=19)$ & $\mathrm{uHC}(n=21)$ & & Group differences & Post-hoc comparisons \\
\hline Age & $25.0(8.0)$ & $30.8(9.5)$ & $31.7(10.6)$ & $25.3(7.5)$ & & $\begin{array}{c}\mathrm{M}: F(1,77)=9.2 \\
p=.003\end{array}$ & $\begin{array}{c}\mathrm{mOCD}>\mathrm{uHC}, \mathrm{uOCD} \\
\mathrm{mPC}>\mathrm{uHC}, \mathrm{uOCD} \\
\text { all } p<.05\end{array}$ \\
\hline Education (years) & $14.9(1.8)$ & $16.1(2.7)$ & $16.8(2.1)$ & $16(2.4)$ & & ns & \\
\hline Gender & $10 \mathrm{~F}, 9 \mathrm{M}$ & $10 \mathrm{~F}, 9 \mathrm{M}$ & $11 \mathrm{~F}, 8 \mathrm{M}$ & $10 \mathrm{~F}, 11 \mathrm{M}$ & & ns & \\
\hline HARS & $8.9(3.8)$ & $9.4(4.8)$ & $4.4(2.7)$ & $1.3(1.6)$ & & $\begin{array}{c}\mathrm{D}: F(1,77)=67.1 \\
p<.001 \\
\mathrm{M}: \begin{array}{c}F(1,77)=5.0 \\
p=.028\end{array}\end{array}$ & $\begin{array}{c}\mathrm{mOCD}>\mathrm{uHC}, \mathrm{mPC} \\
\mathrm{uOCD}>\mathrm{uHC}, \mathrm{mPC} \\
\mathrm{mPC}>\mathrm{uHC} \\
\text { all } p<.001\end{array}$ \\
\hline HDRS & $8.8(2.8)$ & $8.3(3.6)$ & $4.1(2.8)$ & $1.2(1.4)$ & $\mathrm{D}$ & $\begin{array}{c}\mathrm{D}: F(1,77)=92.7 \\
p<.001 \\
\times \mathrm{M}: F(1,77)=7.9 \\
p=.006\end{array}$ & $\begin{array}{c}\mathrm{mOCD}>\mathrm{uHC}, \mathrm{mPC} \\
\mathrm{uOCD}>\mathrm{uHC}, \mathrm{mPC} \\
\mathrm{mPC}>\mathrm{uHC} \\
\text { all } p<.001\end{array}$ \\
\hline History of depression & $10 / 19$ & $15 / 19$ & $19 / 19$ & & & $\begin{array}{c}\chi^{2}(2)=12.2 \\
p=.002\end{array}$ & $\begin{array}{c}\mathrm{mPC}>\mathrm{uOCD} \\
p=.002\end{array}$ \\
\hline YBOCS & $23.4(3.5)$ & $21.6(4.2)$ & & & & ns & \\
\hline Age of onset & $12.5(4.6)$ & $11.7(5.9)$ & & & & ns & \\
\hline Illness duration (years) & $12.5(8.7)$ & $19.1(12.3)$ & & & & ns & \\
\hline In current treatment & $6 / 19$ & $19 / 19$ & & & & $\begin{array}{c}\chi^{2}(1)=19.8 \\
p<.001\end{array}$ & \\
\hline History of hospitalizations & $3 / 19$ & $6 / 19$ & & & & ns & \\
\hline
\end{tabular}

Note: $\mathrm{uOCD}=$ unmedicated $\mathrm{OCD} ; \mathrm{mOCD}=$ medicated $\mathrm{OCD} ; \mathrm{mPC}=$ medicated patient controls; $\mathrm{uHC}=$ unmedicated healthy controls. Values in parentheses represent standard deviations. HARS $=$ Hamilton Anxiety Rating Scale; HDRS $=$ Hamilton Depression Rating Scale; YBOCS $=$ YaleBrown Obsessive Compulsive Scale. $\mathrm{D}=$ main effect of diagnosis factor; $\mathrm{M}=$ main effect of medication factor; $\mathrm{D} \times \mathrm{M}=$ interaction between diagnosis and medication factors. Only those effects significant at $p<.05$ are shown, and followed up with post-hoc comparisons using independentsamples $t$-tests, except history of depression, which used chi-square tests. 
Table 2. List of Medications in Medicated $O C D$ ( $m O C D)$ and Medicated Patient Control (mPC) Groups

\begin{tabular}{|c|c|c|c|c|}
\hline Medications & $\begin{array}{c}N \text { in mOCD } \\
\text { group }\end{array}$ & $\begin{array}{c}\text { Average } \\
\text { dosage (mg) }\end{array}$ & $\begin{array}{c}N \text { in mPC } \\
\text { group }\end{array}$ & $\begin{array}{c}\text { Average } \\
\text { dosage }(\mathrm{mg})\end{array}$ \\
\hline \multicolumn{5}{|l|}{ SSRIs/SNRIs } \\
\hline Citalopram & 3 & 30 & 1 & 40 \\
\hline Escitalopram & 6 & 25 & 9 & 15 \\
\hline Fluoxetine & 4 & 47.5 & 2 & 35 \\
\hline Fluvoxamine & 1 & 250 & & \\
\hline Paroxetine & 1 & 60 & & \\
\hline Sertraline & 2 & 162.5 & 1 & 100 \\
\hline Venlafaxine & 1 & 300 & 6 & 262.5 \\
\hline \multicolumn{5}{|l|}{ Benzodiazepines } \\
\hline Alprazolam & 3 & 0.75 & & \\
\hline Clonazepam & 4 & 0.7 & 2 & 0.7 \\
\hline Lorazepam & 1 & 0.5 & 3 & 0.7 \\
\hline \multicolumn{5}{|l|}{ TCAs } \\
\hline Clomipromine & 1 & 100 & & \\
\hline \multicolumn{5}{|l|}{ Other } \\
\hline Buproprion & 2 & 300 & 2 & 300 \\
\hline Buspirone & & & 1 & 20 \\
\hline Gabapentin & 1 & 600 & & \\
\hline Methylphenidate & & & 1 & 54 \\
\hline
\end{tabular}

Note: SSRIs = selective-serotonin reuptake inhibitors; SNRIs = serotonin-norepinephrine reuptake inhibitors; TCAs $=$ tricycle antidepressants. All subjects with the exception of one mOCD patient were taking a serotonin reuptake inhibitor (SSRI or SNRI). SSRIs were taken by 17/19 mOCDs and 13/19 mPCs, and SNRIs were taken by $1 \mathrm{mOCD}$ and $6 \mathrm{mPC}$ subjects. Ten $\mathrm{mOCD}$ and $8 \mathrm{mPC}$ subjects were taking more than one medication. Benzodiazepines were taken as needed.

(the 6 patients who were in treatment were being seen in our clinic but were not on medication). Despite these differences, the two OCD groups were similar on many of the demographic and clinical measures we examined (see Table 1), although medicated OCD patients were older and had a trend toward longer illness duration $(t(32.4)=1.9, p=.067)$.

Subjects in the mPC group were taking very comparable medications to mOCD (see Table 2) due to a primary diagnosis of major depressive disorder that was in full remission (2 subjects with a history of a single episode and 8 with recurrent episodes) or partial remission ( 9 subjects with recurrent episodes). Importantly, these subjects had no history of OCD and exhibited minimal anxiety comorbidity (5 patients total: 1 with panic disorder, 1 with panic disorder and specific phobia, 1 with specific phobia, and 2 with anxiety disorder NOS), and thus provided partial control for effects related to histories of depressive episodes among OCD patients.

All subjects were evaluated by a trained clinician using the Structured Clinical Interview for DSM-IV (First, Spitzer, Gibbon, \& Williams, 1996). Depression and anxiety symptoms were evaluated using Hamilton Ratings Scales for Depression and Anxiety, respectively, and severity of OC symptoms in OCD patients was measured using the YBOCS.

Written informed consent as approved by the institutional review board of the University of Michigan was obtained from all subjects following a complete description of the study.

\section{Task}

We used a modified version of a flanker task in which subjects pressed one of two buttons based on the identity of a target letter that was placed in the second, third, or fourth position in a string of 5 letters. "Low" interference trials were those where both target and flankers signaled the same button press ("S" and " $\mathrm{K}$ " letters - left button, "H" and "C" letters — right button), while "high" interference trials elicited errors because the target and flankers designated opposing responses. Individually tailored response deadlines, set at $0.8-1.5$ times the mean reaction time (RT) determined from a practice session, were used to generate commission error rates between 10-20\%. Letter stimuli were presented on-screen for $300 \mathrm{~ms}$, followed by a blank screen until the response deadline was achieved. Feedback was presented immediately following response in order to increase motivation to perform well and to ensure that subjects maintained their knowledge of the correct stimulus-response mappings throughout the experiment. A row of white asterisks were presented for correct responses, a row of red asterisks were presented for errors of commission, and a message-" "Too Slow" - was shown if responses were not within the deadline (i.e., omission error). Duration of feedback was 700-1300 ms depending on the individual subject's RT on each trial, so that total time between stimulus presentation and end of feedback presentation was 1800 ms. Following feedback, a blank inter-trial interval (ITI) was shown for $2000 \mathrm{~ms}$.

The task also varied the incentive value of each trial so that an error (or correct response) could result in a loss of money (or a failure to lose), a failure to gain money (or a gain), or no change in money. Cues showing the amount of money at stake $(0,10$, or 50 cents) preceded letter stimuli with durations of $1500-4500 \mathrm{~ms}$ and an average length of $2125 \mathrm{~ms}$ (in order to match a companion fMRI study). As the incentive was not found to have significant effects on the ERN, we focus the current report on group effects on the overall ERN. Money won or lost on each trial was real, and tallied to provide a bonus at the end of the experiment. A total of 480 trials (240 low and 240 high interference) were run over 10 blocks.

In order to determine how subjects evaluated the task and their performance after completing the experiment, ratings to three debriefing questions were obtained on a five-point Likert scale ranging from "none/not at all" (1) to "always/very" (5): 1) "Did you make any mistakes?," 2) "Were you ever frustrated with your performance?," and 3) "When you made a mistake, were you flustered? Did you find it hard to get back on track?"

\section{Data Acquisition and Analysis}

Behavioral analyses examined RT on correct trials, commission error rates, and responses to debriefing questions as dependent measures in separate $2 \times 2$ ANOVAs using diagnosis (OCD vs. control) and medication status (unmedicated vs. medicated) as between-subjects factors. Omission errors were excluded from all analyses.

The EEG was recorded from $26 \mathrm{Ag} / \mathrm{AgCl}$ scalp electrodes (FP1, FP2, AFZ, F7, F3, FZ, F4, F8, FC3, FCZ, FC4, T7, C3, $\mathrm{CZ}, \mathrm{C} 4, \mathrm{~T} 8, \mathrm{CP} 3, \mathrm{CPZ}, \mathrm{CP} 4, \mathrm{P} 7, \mathrm{P} 3, \mathrm{PZ}, \mathrm{P} 4, \mathrm{P} 8, \mathrm{O} 1, \mathrm{O} 2) \mathrm{em}-$ bedded in a nylon mesh cap (Easy-Cap, Falk Minow Systems, Inc., http://www.easycap.de) using a left mastoid reference and forehead ground. Average mastoid reference was derived off-line using right mastoid data. The electro-oculogram (EOG) was recorded from $\mathrm{Ag} / \mathrm{AgCl}$ electrodes above and below the left eye and external to the outer canthus of each eye. Impedances were kept below $10 \mathrm{~K} \Omega$. EEG and EOG were amplified by SYNAMPS DC amplifiers (Neuroscan Labs, Sterling, VA) and filtered on-line from .01 to $100 \mathrm{~Hz}$ (half-amplitude cutoffs). Data were digitized at $500 \mathrm{~Hz}$ and filtered with a nine-point Chebyshev II low-pass, zero-phase-shift digital filter (Matlab 7.04; 
Mathworks, Natick, MA), half-amplitude cutoff at $12 \mathrm{~Hz}$. Ocular movement artifacts were corrected using the algorithm described by Gratton, Coles, and Donchin (1983).

In order to correct for the fact that the response-locked ERN will actually reflect some combination of response and stimulusevoked components, we applied a method (Zhang, 1998) that allows for the unique recovery of the response-locked component based on the stimulus-locked ERP, response-locked ERP, and the time between stimulus and response on each trial (reaction time distribution). The method works as follows. We have experimentally obtained (a) stimulus-aligned ERP average waveform, denoted $F_{s}(t)$; (b) response-aligned ERP average waveform, denoted $F_{r}(t)$; and (c) distribution of reaction times $g(t)$ across the trials. Suppose the stimulus-aligned and responsealigned ERP waveforms were generated by two underlying component waveforms, a stimulus-locked component ("S-component"), denoted $f_{s}(t)$, and a response-locked component ("Rcomponent"), denoted $f_{r}(t)$. By reflecting on how $F_{s}(t)$ and $F_{r}(t)$ were constructed and how $f_{s}(t)$ and $f_{r}(t)$ were defined, the following two mathematical equations were derived:

$$
\begin{aligned}
& F_{S}(t)=f_{s}(t)+\int f_{r}(t-\tau) g(\tau) d \tau \\
& F_{r}(t)=f_{r}(t)+\int f_{s}(t+\tau) g(\tau) d \tau
\end{aligned}
$$

In convolution notation, they are

$$
\begin{gathered}
F_{S}(t)=f_{s}(t)+f_{r}(t) * g(t) \\
F_{r}(t)=f_{r}(t)+f_{s}(t) * g(-t)
\end{gathered}
$$

We can then solve these equations (3) and (4) either by Fourier transformation (in frequency domain) or by an iterative procedure (in time domain). In practice, due to discrete sampling in time domain, we can transform equation (3) and (4) into matrix notation (Yin, Zhang, Tian, \& Yao, 2009), and apply singular value decomposition (SVD) method to the reaction-time distribution to deal with noise introduced by insufficient number of trials. In this paper, the time domain SVD method (same as Fourier transform method in nature) was used, by removing/ truncating small eigenvalues (of the reaction time distribution matrix) to reduce the influence of noise and improve stability.

After recovery of the response-locked waveform, mean amplitude was calculated over a window of $20-120$ ms post-response (identified from grand-averaged waveforms) with a baseline of 200 to $100 \mathrm{~ms}$ prior to response. Three-way ANOVAs with diagnosis (OCD vs. control) and medication status (unmedicated vs. medicated) as between-subjects factors and trial type (error vs. correct) as a within-subjects factor were performed separately at electrodes FZ and FCZ. Analyses were performed separately at these two electrodes because inspection of the topographical distribution of scalp activity indicated that the peak of the ERN was located at FCZ for control subjects but anterior to FCZ for OCD patients (see Figure 1). Pearson's correlations (two-tailed) of error and correct trial amplitudes with anxiety (HARS) and depression (HDRS), and for OCD patients, YBOCS scores, were used to further interrogate effects identified by the ANOVAs. The mean number of errors per subject contributing to the analysis was 63.94 (SD: 32.77, range: 15-150).

\section{Results}

\section{Behavioral}

There were no significant differences between the groups for mean RT on correct trials, although there was a trend for an interaction between diagnosis and medication $(F(1,74)=3.3$, $p=.072)$, with unmedicated healthy controls exhibiting the fastest RTs and medicated patient controls exhibiting the slowest RTs (uOCD: 569.2, mOCD: 566.7, mPC: 597.8, uHC: $532.7 \mathrm{~ms}$ ). Similarly, there were no differences in the mean percentage of commission errors between groups (uOCD: $12.5 \%$, mOCD: 14\%, mPC: $12.9 \%$, uHC: $13.5 \%$ ).

Analyses of responses to debriefing questions indicated no main effects or interactions of group factors on subjects' evaluation of the amount of mistakes that were made ( $p>.25$ for all). Interestingly, despite similar performance accuracy, OCD patients reported being significantly more frustrated with their performance $(F(1,74)=22.1, p<.001)$ and more flustered when making a mistake $(F(1,74)=21.9, p<.001)$ than control subjects. No other effects were found, with the exception of a trend toward unmedicated subjects being slightly more flustered when making a mistake than medicated subjects $(F(1,74)=3.3$, $p=.073)$.

\section{Electrophysiological}

As expected, there was a highly significant main effect of trial type (error vs. correct) at both electrodes (FZ: $F(1,74)=175$, $p<.001$; FCZ: $F(1,74)=199.9, p<.001)$, such that amplitudes were more negative for errors as compared to correct trials. There were no main effects of group factors (diagnosis or medication status); however, there was a significant interaction between trial type and diagnosis (OCD vs. control) at electrode FZ ( $F(1$, $74)=4.1, p=.047)$, indicating that OCD patients exhibited a greater ERN than control subjects $(-4.0$ vs. $-2.5 \mu \mathrm{V}$, $t(76)=-1.98, p=.05)$, with no difference in amplitude on correct trials $(2.7 \text { vs. } 2.4 \mu \mathrm{V}, t(76)=0.38, p=.71)^{1}$ (Figures 1 and 2). Importantly, there were no 2-way interactions between trial type and medication status or 3-way interactions involving trial type, medication status, and diagnosis (all $p \mathrm{~s}>0.6$ ), indicating that the presence of chronic medication did not significantly influence the ERN. No effects of group factors were found at electrode FCZ.

Among OCD patients, ERN amplitudes were not significantly correlated with YBOCS scores $(r=.17, p=.32)$ or with generalized anxiety or depressive symptoms (HARS: $r=.13$, $p=.43$, HDRS: $r=.10, p=.57$ ) (Figure 3). By contrast, among control subjects, there was a significant correlation between ERN amplitude and HARS $(r=-.35, p=.027)$ and HDRS $(r=-.35, p=.026)$, such that greater symptom severity was associated with an increased ERN. As can be seen from Figure 3, these relationships were found within both $\mathrm{uHC}$ and $\mathrm{mPC}$ groups for HARS $(r=-.49, p=.023$ and $r=-.41, p=.082$, respectively) and HDRS $(r=-.39, p=.081$, and $r=-.46$, $p=.050$, respectively) scores. Correct trial amplitudes were not

${ }^{1}$ When removing the one mOCD patient not taking a serotonin reuptake inhibitor (i.e., buproprion), the interaction remained significant, $F(1,73)=7, p=.034$. 


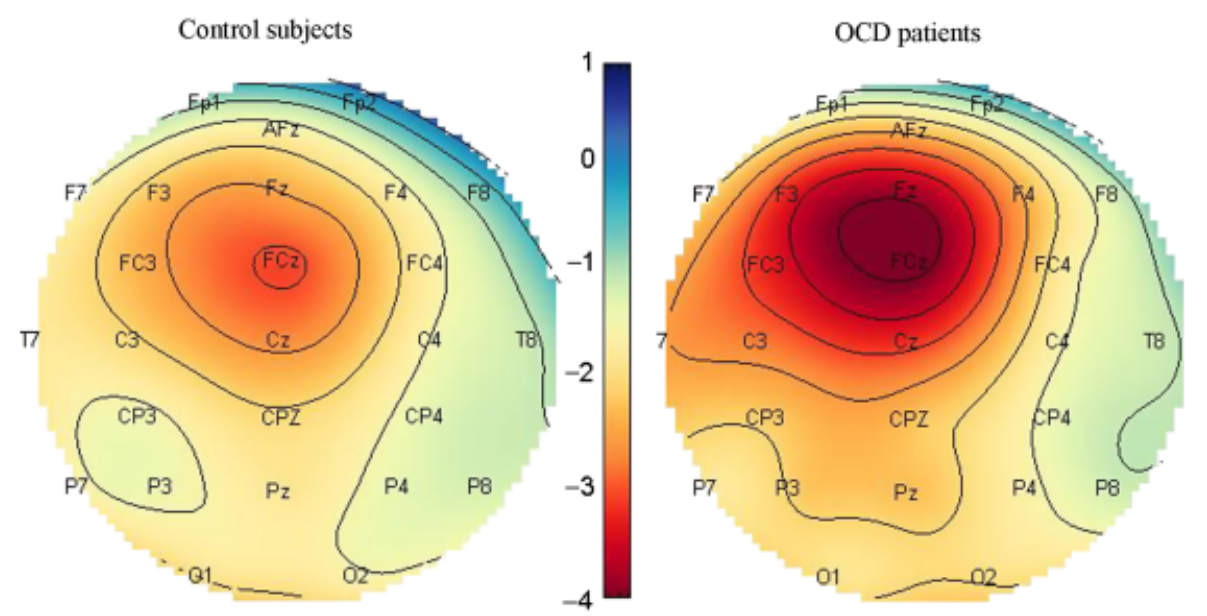

Figure 1. Topographic maps of ERN amplitude. Error activity shows a medial frontal focus, with the peak of activity in OCD patients located slightly anterior to that of control subjects. Scale represents mean activity in $\mu \mathrm{V}$ between 20 and $120 \mathrm{~ms}$ post error response.

correlated with symptom severity measures for either OCD patients or controls.

\section{Discussion}

Previous studies have identified an increased ERN in patients with OCD (Endrass et al., 2008; Gehring et al., 2000; Johannes et al., 2001), yet it has been unclear whether the use of serotonin reuptake inhibitors among OCD patients may be influencing this finding given evidence linking serotonergic functioning to the ERN (Fallgatter et al., 2004; Jocham \& Ullsperger, 2009). Methodologically, it is difficult to separate primary markers of a disorder from secondary phenomena related to treatment effects and comorbidity. As such, it may be particularly advantageous to compare treated and untreated patient groups matched on symptom severity, along with a treated "control" group with a diagnosis that is highly comorbid with the primary disorder, as was done in the current investigation. Our results indicated that OCD patients exhibited an increased ERN irrespective of medication use, and that medication use in patient controls was not associated with any elevation in the ERN compared to healthy controls. This extends previous findings of no effect of acute administration of selective-serotonin reuptake inhibitor (SSRI) on the ERN in healthy controls (de Bruijn, Sabbe, Hulstijn, Ruigt, \& Verkes, 2006) to include chronic administration of SRIs in a patient population. The current findings suggest that ERN hyperactivity in OCD is not an epiphenomenon of SSRI administration, and that if serotonergic activity does impact the ERN (Fallgatter et al., 2004; Jocham \& Ullsperger 2009), it may not be a simple effect of reuptake inhibition.

Does an increased ERN simply reflect greater anxiety and/or depression in OCD patients rather than being specific to OCD? Previous studies have indeed found greater ERNs associated with generalized anxiety (Hajcak, McDonald, \& Simons, 2003) and major depressive disorder (Chiu \& Deldin, 2007; Holmes \& Pizzagalli, 2008). Our results indicated that, while OCD patients exhibited more anxiety and depressive symptoms (in addition to OC symptoms) than either control group, their level of these symptoms did not predict ERN amplitude. Intriguingly, within both control groups, greater anxiety and depression were associated with increased ERN amplitude. It is possible that the ERN is normally influenced by levels of negative affect, but that
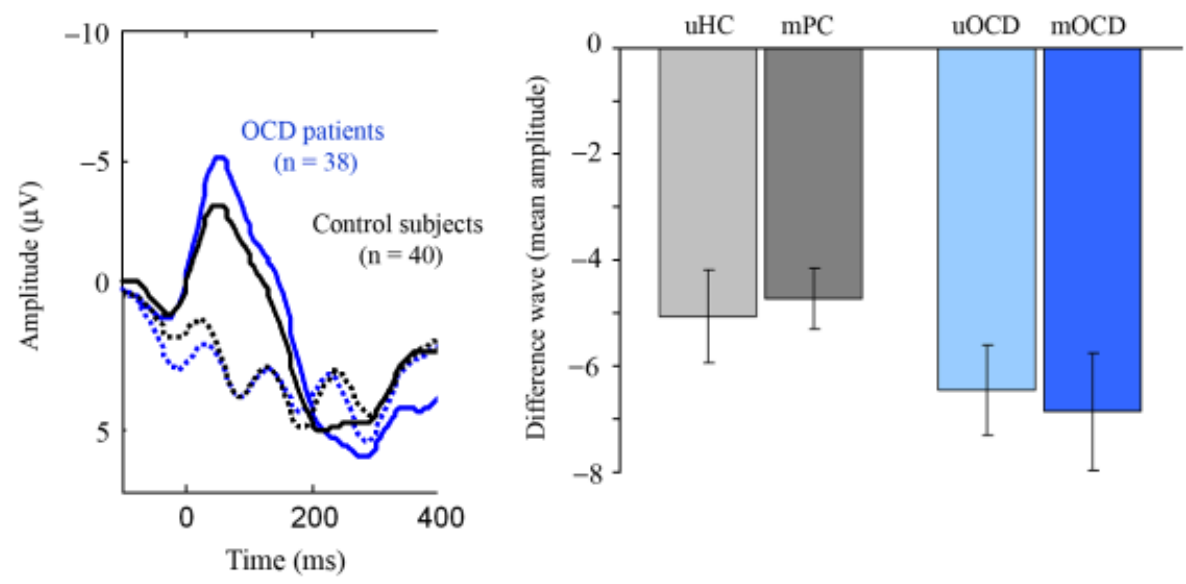

Figure 2. Error and correct trial waveforms for OCD patients and control subjects. OCD patients (blue lines) exhibited greater amplitude for errors (solid) but not correct trials (dashed) as compared to control subjects (black lines). Bar graph shows the amplitude of the difference wave (error-correct) in each group. uHC = unmedicated healthy controls; $\mathrm{mPC}=$ medicated patient controls; uOCD $=$ unmedicated $\mathrm{OCD}$ patients; $\mathrm{mOCD}=$ medicated OCD patients. 
- unmedicated healthy controls

- medicated patient controls
- unmedicated OCD

- medicated OCD

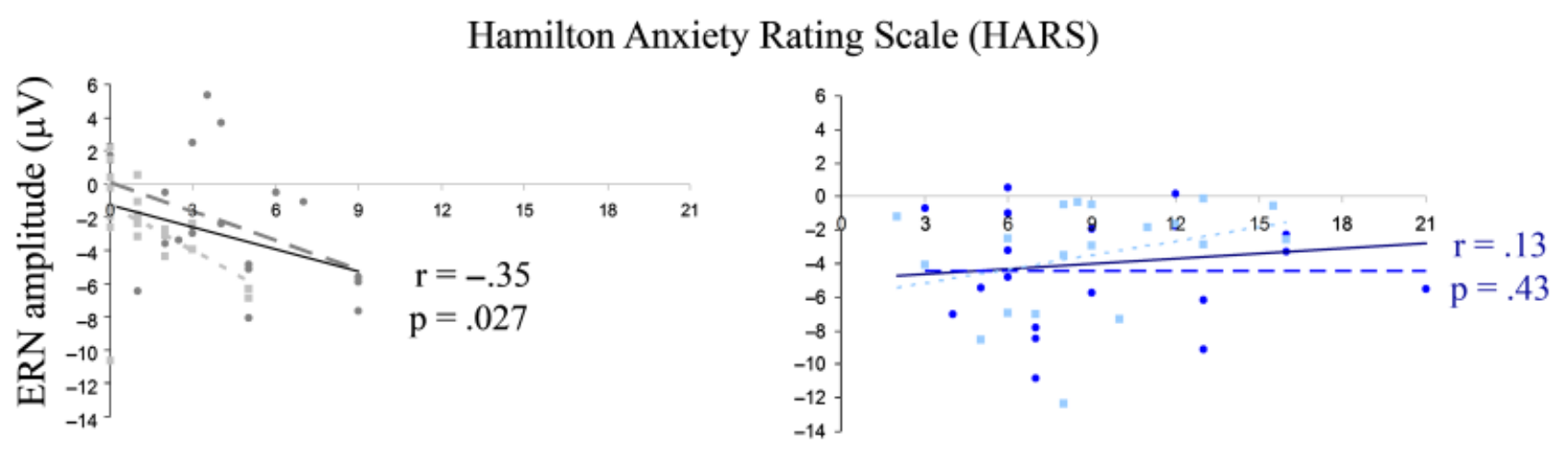

Hamilton Depression Rating Scale (HDRS)
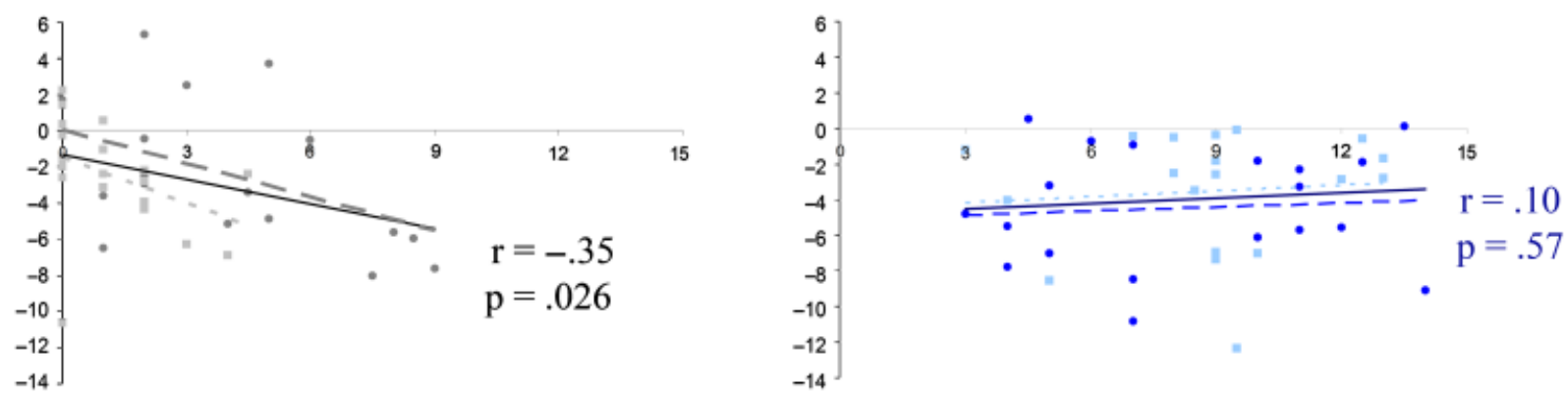

Figure 3. Correlations between ERN amplitude and symptoms of generalized anxiety and depression. Significant relationships were found in the control group but not in the OCD group. Trend lines are shown for correlations in all control subjects (solid black) and all OCD patients (solid blue), and for each group separately (unmedicated healthy controls: short dashed light gray; medicated patient controls: long dashed dark gray; unmedicated OCD patients: short dashed light blue; medicated OCD patients: long dashed dark blue).

in OCD this mechanism is overshadowed by a disorder-specific abnormality that is not related to the severity of generalized anxiety or depressive symptoms.

Although symptoms of depression were related to an increased ERN in unmedicated healthy and medicated patient controls, a diagnosis of major depressive disorder in remission was not associated with an overall enhancement of the ERN in the mPC group. Such a distinction between effects of current versus prior depressive symptomatology on the ERN may help explain inconsistencies among previous studies examining the ERN in depression, which have identified no differences between remitted depressed patients and controls (Ruchsow et al., 2004, 2006), but an increased ERN in moderately depressed patients in a current depressive episode (Chiu \& Deldin, 2007; Holmes \& Pizzagalli, 2008).

Our data indicated that ERNs were similarly increased for medicated and unmedicated OCD groups, which was unexpected given previous findings of a reduction in cingulate and orbitofrontal hyperactivity after a course of medication in OCD patients (Perani et al., 1995; Saxena et al., 1999). One possibility is that the medicated group was actually more severely ill prior to their initiation of treatment, and would have shown greater YBOCS scores and ERNs than unmedicated patients had they been tested in an untreated state. However, as ERN amplitudes were not correlated with YBOCS scores in the OCD group, our data do not support the hypothesis that the increased ERN found in OCD is a function of OC symptom severity. Rather, this result provides support for the notion that ERN hyperactivity may be a trait marker of OCD that is not sensitive to fluctuations in symptom severity and does not decrease in response to treatment, consistent with a recent study reporting no change in ERN hyperactivity among children with OCD after treatment with cognitive behavioral therapy (Hajcak, Franklin, Foa, \& Simons, 2008).

While the ERN was robust for all groups at electrodes FZ and FCZ, the difference between OCD patients and controls was greatest at the more anterior electrode FZ. This is likely due to the slightly anterior topography of the ERN in OCD patients as compared to control subjects, which may have psychological significance. Although the ERN is typically thought to have a source in posterior/dorsal regions of medial frontal cortex/anterior cingulate gyrus (Dehaene et al., 1994; van Veen \& Carter, 2002), it has also been shown to correlate with activity in anterior/rostral regions of medial frontal cortex (Mathalon, Whitfield, \& Ford, 2003). Anterior MFC is involved in emotion and social processing (Amodio \& Frith, 2006; Bush, Luu, \& Posner, 2000; Steele \& Lawrie, 2004), and it stands to reason that the relative contribution of posterior versus anterior regions of MFC to the ERN may, in fact, vary based upon the psychological reaction to making an error. In our study, OCD patients were 
more frustrated with their performance and more flustered when making an error than control subjects. It is possible that the emergence of the group difference at the more anterior site is due to a greater contribution of anterior regions of MFC to the ERN in OCD patients, in relation to their heightened emotional reaction. Although speculative, such a hypothesis is consistent with previous fMRI data identifying error-related hyperactivation of anterior MFC in OCD patients (Fitzgerald et al., 2005).

There are several limitations to the current study that suggest avenues for future research. First, performance feedback provided on a trial-by-trial basis was used to increase motivation, but may have reduced the response-locked signal by shifting attention away from the response toward the feedback. As it is conceivable that error feedback would be more salient, and hence divert more attention, than correct feedback, this feature of the study may have lead to a disproportionate reduction in the ERN compared to correct trial ERPs. However, given that there were no differences in accuracy between groups, this is unlikely to account for the greater ERN in OCD patients. Second, although mPCs and mOCDs were nearly all taking some form of SRI medication, we did not control for the concomitant use of benzodiazepines or non-SRI antidepressant medication. The medicated groups had similar proportions of subjects taking these additional medications, however, and greater use of benzodiazepines among OCD patients cannot explain their exaggerated ERN relative to controls, since benzodiazepines have been associated with a reduced ERN (de Bruijn, Hulstijn, Verkes, Ruigt,
\& Sabbe, 2004; Riba, Rodríguez-Fornells, Münte, \& Barbanoj, 2005). Third, among SRI types, there was a greater proportion of SNRIs being taken by mPCs than mOCD patients. Yet, as norepinephrine release has been shown to increase the ERN (Riba, Rodríguez-Fornells, Morte, Münte, \& Barbanoj, 2005), this, too, would work against the finding of exaggerated the ERN in the OCD patients. Sample sizes were too small to segregate medicated subjects based on SSRI, SNRI, and additional medication use, so more detailed analysis of medication effects on the ERN will require replication with a larger sample. Subjects included here were not taking antipsychotic medications, but considering that these are sometimes used as adjunctive therapy in OCD and can reduce the ERN (de Bruijn et al., 2006), future studies should seek to track or exclude their use as well.

In sum, our results indicate that OCD patients have exaggerated ERNs that are not due to SRI medication use. The data also show that greater severity of generalized anxiety and depressive symptoms is associated with increased ERNs among control subjects, but that these symptoms do not account for ERN hyperactivity in OCD patients. Instead, our results suggest that an increased ERN may be a neural endophenotype of OCD that does not fluctuate with symptom severity or decrease with pharmacological treatment. Future studies would benefit from comparing trait and state influences on ERN hyperactivity across different psychiatric disorders, and by examining genetic influences on the ERN in OCD, in order to further explore these effects.

\section{REFERENCES}

Amodio, D. M., \& Frith, C. D. (2006). Meeting of minds: The medial frontal cortex and social cognition. Nature Reviews Neuroscience, 7 , 268-277.

Bush, G., Luu, P., \& Posner, M. I. (2000). Cognitive and emotional influences in anterior cingulate cortex. Trends in Cognitive Sciences, 4 , 215-222.

Chiu, P. H., \& Deldin, P. J. (2007). Neural evidence for enhanced error detection in major depressive disorder. American Journal of Psychiatry, 164, 608-616.

de Bruijn, E. R. A., Hulstijn, W., Verkes, R. J., Ruigt, G. S. F., \& Sabbe, B. G. C. (2004). Drug-induced stimulation and suppression of action monitoring in healthy volunteers. Psychopharmacology, 177, 151160 .

de Bruijn, E. R. A., Sabbe, B. G. C., Hulstijn, W., Ruigt, G. S. F., \& Verkes, R. J. (2006). Effects of antipsychotic and antidepressant drugs on action monitoring in healthy volunteers. Brain Research, 1105, 122-129.

Dehaene, S., Posner, M. I., \& Tucker, D. M. (1994). Localization of a neural system for error detection and compensation. Psychological Science, 5, 303-305.

Endrass, T., Klawohn, J., Schuster, F., \& Kathmann, N. (2008). Overactive performance monitoring in obsessive-compulsive disorder: ERP evidence from correct and erroneous reactions. $\mathrm{Ne}$ uropsychologia, 46, 1877-1887.

Falkenstein, M., Hohnsbein, J., Hoormann, J., \& Blanke, L. (1990). Effects of errors in choice reaction tasks on the ERP under focused and divided attention. In C. Brunia, A. Gaillard, \& A. Kok (Eds.), Psychophysiological brain research (pp. 192-195). The Netherlands: Tilburg University Press.

Fallgatter, A. J., Herrmann, M. J., Roemmler, J., Ehlis, A.-C., Wagener, A., Heidrich, A., et al. (2004). Allelic variation of serotonin transporter function modulates the brain electrical response for error processing. Neuropsychopharmacology, 29, 1506-1511.

First, M., Spitzer, R., Gibbon, M., \& Williams, J. (1996). Structured clinical interview for DSM-IV axis disorders (SCID), clinician version: User's guide. Washington, DC: American Psychiatric Press.

Fitzgerald, K. D., Welsh, R. C., Gehring, W. J., Abelson, J. L., Himle, J. A., Liberzon, I., \& Taylor, S. F. (2005). Error-related hyperactivity of the anterior cingulate cortex in obsessive-compulsive disorder. Biological Psychiatry, 57, 287-294.

Gehring, W. J., Goss, B., Coles, M. G., Meyer, D. E., \& Donchin, E. (1993). A neural system for error detection and compensation. Psychological Science, 4, 385-390.

Gehring, W. J., Himle, J., \& Nisenson, L. G. (2000). Action-monitoring dysfunction in obsessive-compulsive disorder. Psychological Science, $11,1-6$.

Goodman, W. K., Price, L. H., Rasmussen, S. A., Mazure, C., Fleischmann, R. L., Hill, C. L., et al. (1989). The Yale-Brown Obsessive Compulsive Scale. I. Development, use, and reliability. Archives of General Psychiatry, 46, 1006-1011.

Gratton, G., Coles, M. G., \& Donchin, E. (1983). A new method for offline removal of ocular artifact. Electroencephalography and Clinical Neurophysiology, 55, 468-484.

Hajcak, G., Franklin, M. E., Foa, E. B., \& Simons, R. F. (2008). Increased error-related brain activity in pediatric obsessive-compulsive disorder before and after treatment. American Journal of Psychiatry, $165,116-123$.

Hajcak, G., McDonald, N., \& Simons, R. F. (2003). Anxiety and errorrelated brain activity. Biological Psychology, 64, 77-90.

Hajcak, G., \& Simons, R. F. (2002). Error-related brain activity in obsessive-compulsive undergraduates. Psychiatry Research, 110, 63-72.

Holmes, A. J., \& Pizzagalli, D. A. (2008). Spatiotemporal dynamics of error processing dysfunctions in major depressive disorder. Archives of General Psychiatry, 65, 179-188.

Holroyd, C. B., \& Coles, M. G. H. (2002). The neural basis of human error processing: Reinforcement learning, dopamine, and the errorrelated negativity. Psychological Review, 109, 679-709.

Jocham, G., \& Ullsperger, M. (2009). Neuropharmacology of performance monitoring. Neuroscience and Biobehavioral Reviews, 33, 4860.

Johannes, S., Wieringa, B. M., Nager, W., Rada, D., Dengler, R., Emrich, H. M., et al. (2001). Discrepant target detection and action monitoring in obsessive-compulsive disorder. Psychiatry Research, $108,101-110$

Mathalon, D. H., Whitfield, S. L., \& Ford, J. M. (2003). Anatomy of an error: ERP and fMRI. Biological Psychology, 64, 119-141. 
Nieuwenhuis, S., Nielen, M. M., Mol, N., Hajcak, G., \& Veltman, D. J. (2005). Performance monitoring in obsessive-compulsive disorder. Psychiatry Research, 134, 111-122.

Olvet, D. M., \& Hajcak, G. (2009). The stability of error-related brain activity with increasing trials. Psychophysiology, 46, 957-961.

Perani, D., Colombo, C., Bressi, S., Bonfanti, A., Grassi, F., Scarone, S., et al. (1995). [18F]FDG PET study in obsessive-compulsive disorder. A clinical/metabolic correlation study after treatment. British Journal of Psychiatry, 166, 244-250.

Pitman, R. K. (1987). A cybernetic model of obsessive-compulsive psychopathology. Comprehensive Psychiatry, 28, 334-343.

Riba, J., Rodríguez-Fornells, A., Morte, A., Münte, T. F., \& Barbanoj, M. J. (2005). Noradrenergic stimulation enhances human action monitoring. Journal of Neuroscience, 25, 4370-4374.

Riba, J., Rodríguez-Fornells, A., Münte, T. F., \& Barbanoj, M. J. (2005). A neurophysiological study of the detrimental effects of alprazolam on human action monitoring. Brain Research. Cognitive Brain Research, 25, 554-565.

Ruchsow, M., Herrnberger, B., Beschoner, P., Grön, G., Spitzer, M., \& Kiefer, M. (2006). Error processing in major depressive disorder: Evidence from event-related potentials. Journal of Psychiatric Research, 40, 37-46.

Ruchsow, M., Herrnberger, B., Wiesend, C., Grön, G., Spitzer, M., \& Kiefer, M. (2004). The effect of erroneous responses on response monitoring in patients with major depressive disorder: A study with event-related potentials. Psychophysiology, 41, 833-840.

Saxena, S., Brody, A. L., Maidment, K. M., Dunkin, J. J., Colgan, M., Alborzian, S., et al. (1999). Localized orbitofrontal and subcortical metabolic changes and predictors of response to paroxetine treatment in obsessive-compulsive disorder. Neuropsychopharmacology, 21, 683-693.

Schwartz, J. (1997). Obsessive-compulsive disorder. Science \& Medicine, $2,14-23$.

Steele, J. D., \& Lawrie, S. M. (2004). Segregation of cognitive and emotional function in the prefrontal cortex: A stereotactic meta-analysis. NeuroImage, 21, 868-875.

Taylor, S. F., Stern, E. R., \& Gehring, W. J. (2007). Neural systems for error monitoring: Recent findings and theoretical perspectives. $\mathrm{Ne}$ uroscientist, 13, 160-172.

Ursu, S., Stenger, V. A., Shear, M. K., Jones, M. R., \& Carter, C. S. (2003). Overactive action monitoring in obsessive-compulsive disorder: Evidence from functional magnetic resonance imaging. Psychological Science, 14, 347-353.

van Veen, V., \& Carter, C. S. (2002). The anterior cingulate as a conflict monitor: fMRI and ERP studies. Physiology and Behavior, 77, 477482.

Yin, G., Zhang, J., Tian, Y., \& Yao, D. (2009). A multi-component decomposition algorithm for event-related potentials. Journal of Neuroscience Methods, 178, 219-227.

Zhang, J. (1998). Decomposing stimulus and response component waveforms in ERP. Journal of Neuroscience Methods, 80, 49-63.

(Received September 22, 2009; ACCEPTED September 23, 2009) 